Dialéctica: método y sujeto. Notas en torno a la obra de Rubén Dri

Martin Retamozo

Tram[p]as de la comunicación y la cultura (N. $\left.{ }^{\circ} 85\right)$, e043, 2020

ISSN 2314-274X | https://doi.org/10.24215/2314274xe043

http://perio.unlp.edu.ar/ojs/index.php/trampas

FPyCS | Universidad Nacional de La Plata

La Plata | Buenos Aires | Argentina

\title{
DIALÉCTICA: MÉTODO Y SUJETO
}

\section{NOTAS EN TORNO A LA OBRA DE RUBÉN DRI}

DIALECTIC: METHOD AND SUBJECT. NOTES ABOUT THE WORK OF RUBEN DRI

\author{
Martin Retamozo \\ martin.retamozo@gmail.com \\ https://orcid.org/0000-0001-8778-7667 \\ Instituto de Investigaciones en Humanidades y Ciencias Sociales (IdIHCS) \\ Facultad de Humanidades y Ciencias de la Educación \\ Universidad Nacional de La Plata | Argentina
}

\begin{abstract}
Resumen
Rubén Dri es uno de los pensadores excepcionales de nuestro tiempo y uno de los más rigurosos conocedores de la obra de Hegel. En el marco de la concepción de la dialéctica hegeliana y de su influencia en el pensamiento de Marx que presenta Dri, el artículo se enfoca en dos aspectos centrales para el pensamiento político contemporáneo: la cuestión del método y el problema del sujeto. El tratamiento de estas cuestiones aporta al debate sobre el lugar de la dialéctica en la teoría crítica y contribuye a establecer bases para indagar sobre estos aspectos en clave contemporánea.
\end{abstract}

Abstract

Rubén Dri is one of the exceptional thinkers of our time and one of the most rigorous experts on Hegel's work. Within the framework of Dri's conception of Hegelian dialectics and its influence on Marx's thought, we propose to focus on two central aspects for contemporary political thought: the question of method and the problem of the subject. The approach to these questions contributes to the debate on the place of dialectics in critical theory and helps to establish a basis for investigating these aspects in a contemporary key.

Palabras clave | Rubén Dri, Hegel, Marx, dialéctica

Keywords | Rubén Dri, Hegel, Marx, dialectic

Recibido: 12/12/2019 | Aceptado: 14/04/2020 | Publicado: 11/08/2020 


\title{
DIALÉCTICA: MÉTODO Y SUJETO \\ NOTAS EN TORNO A LA OBRA DE RUBÉN DRI
}

\author{
Por Martin Retamozo
}

Megafón usaba un método bárbaro
que consistía en buscar solo
aquellas nociones que sirviesen
a su problemática interna.
Leopoldo Marechal (1970)

\section{Introducción}

Rubén Dri (Federación, Argentina, 1929) es uno de los pensadores excepcionales de nuestro tiempo, además de un militante incansable que transita, con la misma humildad y rigurosidad, las universidades, las unidades básicas y las capillas de barrio. Ordenado sacerdote y formado en teología, Dri participó del Movimiento de Sacerdotes para el Tercer Mundo y de organizaciones revolucionarias de los años sesenta y setenta como las Fuerzas Armadas Peronistas y el Peronismo de Base. Luego de su paso a la clandestinidad, su exilio en México y su regreso al país como profesor de la Universidad de Buenos Aires (UBA), Rubén articuló la militancia en distintos espacios con un sistemático estudio de la filosofia de Hegel, lo que permite ubicarlo, en la actualidad, como una de las principales referencias sobre el filósofo alemán en América Latina.

Este trabajo surge a partir de la publicación de su último libro, El movimiento dialéctico: de la Fenomenología del Espíritu de Hegel a los Grundrisse de Marx (Biblos, 2019). ${ }^{1}$ Mediante la recuperación de los interrogantes y los aportes de su obra, buscamos plantear algunos ejes que ligan dos nudos problemáticos en torno al método y a los sujetos con uno de los conceptos más debatidos en la tradición marxista: la dialéctica. 
En esta obra, Dri (2019) pretende indagar en esa intrincada y polémica relación entre Friedrich Hegel y Karl Marx. Como recuerda este pensador, según Vladimir Lenin: "Es completamente imposible entender El capital de Marx, y en especial su primer capítulo, sin haber estudiado y entendido a fondo toda la Lógica de Hegel. ¡Por consiguiente, hace medio siglo ninguno de los marxistas entendía a Marx!» (Dri, 2019, pp. 11-12). Precisamente, toda la obra de nuestro autor y, especialmente, su último libro, busca aportar claridad a esta relación a partir de una hipótesis fuerte de lectura.

El desafío de trabajar el vínculo Hegel-Marx requiere de descomponerlo como problema de investigación (en un momento analítico propio del entendimiento) para reconstruirlo (en un momento sintético propio de la razón). Esto implica llevar a cabo, al menos, cuatro tareas. La primera, comprender qué dijo Hegel a partir de una hipótesis interpretativa exegética; la segunda -en el mismo sentido-, precisar qué afirmó Marx sobre temas que también versó Hegel. La tercera, interrogarse por la influencia de Hegel en Marx, tanto directamente como por un ambiente poshegeliano y, especialmente, a partir de los trabajos de Ludwig A. Feuerbach. La cuarta y última, establecer qué nos puede aportar a nosotros/as, lectores/as del siglo XXI, la profundización en el estudio de esta relación en un horizonte emancipatorio. Este artículo se propone escudriñar estas cuestiones atendiendo a las cuatro tareas mencionadas para abordar los dos nudos problemáticos identificados: la cuestión del método y la cuestión del sujeto.

\section{Hegel y Marx}

Todos sabemos que Hegel es un autor dificil para entrar en soledad. Si, como decía Jorge Luis Borges (1974), "todo lenguaje es un alfabeto de símbolos cuyo ejercicio presupone un pasado que los interlocutores comparten" (p. 624), en el caso de Hegel ese lenguaje es el de la filosofia alemana y la construcción de una jerga propia en un momento cruzado, exacto y dislocado entre la Ilustración y el Romanticismo. Leer, entonces, términos como conciencia, espiritu y religión sin su contexto puede conducir a extravios y a rápidos juicios condenatorios. Pero Hegel mismo nos ofrece una clave de lectura, una sugerencia metodológica como todo gran profesor (cabe recordar que Hegel fue 
durante mucho tiempo director de un gymnasium -una escuela secundariaen Nuremberg): el lugar de la intersubjetividad. A Hegel hay que leerlo conotros. En este sentido, la secuencia de libros de Dri ofrece un itinerario a partir de diferentes obras o pasajes de Hegel. Entre ellos se destacan: Razón y libertad. Hermenéutica del capítulo V de la Fenomenología del espíritu (1994); La odisea de la conciencia moderna. Hermenéutica del capitulo VI de la Fenomenología del espiritu (1998); Racionalidad, sujeto y poder. Irradiaciones de Fenomenología del espíritu (2002); La Fenomenología del espíritu de Hegel. Perspectiva latinoamericana. Intersubjetividad y reino de la verdad (2006); Hegel y la lógica de la liberación. La dialéctica del sujeto-objeto (2007); La rosa en la cruz. La filosofía politica hegeliana (2009); Hegelianas. Irradiaciones de la Fenomenología del espiritu (2011).

Este conjunto de obras contribuye en tres aspectos. Primero, porque contextualiza los escritos de Hegel en un doble sentido: para la exégesis y para la hermenéutica. Para la exégesis, ya que repone los debates que desarrollaba Hegel en su tiempo. Para la hermenéutica, porque nos ofrece claves para un Hegel (y un Marx) aquí y ahora. En efecto, no se trata de una búsqueda meramente erudita (y vaya si lo es la obra de Dri) sino profundamente política. Segundo, porque clarifica los movimientos conceptuales de Hegel y su recepción en Marx, uno de los temas más discutidos tanto en la filosofia política como en la teoría social. Los debates de Marx en un terreno dominado por el joven hegelianismo, su lectura de la Filosofia del Derecho, su reencuentro con la Lógica de Hegel en el invierno de 1858 y su indignación cuando trataban a Hegel de perro muerto -impulso que lo llevó, según sus palabras, a declararse discípulo de Hegel-, han sido objeto de múltiples interpretaciones. Tercero, por lo que nos dice esa relación en el siglo XXI.

Esta tríada estuvo presente en el profundo y beligerante debate sobre la influencia de Hegel sobre Marx. Por un lado, en el marco de una serie de intentos de un Marx deshegelianizado (en ocasiones más kantiano o spinoziano) como es el austromarxismo (en la pluma de Max Adler, por ejemplo) y la escuela dellavolpiana en Italia. Por otro, a partir de una recepción que ponderó positivamente la influencia de Hegel en el horizonte marxista. Obviamente, podemos invocar a grandes pensadores y militantes como Antonio Gramsci, György Lukács, Jean Paul Sartre, Ernst Bloch y Karel Kosik. Pero, además, nos resulta clave -y mucho menos explorada- la influencia 
de Hegel en el pensamiento nacional que se tensiona con el marxismo. Cabe recordar a Carlos Astrada (quien estudió con Edmund Husserl y con Martin Heidegger) o a Juan José Hernández Arregui (que estudió con Rodolfo Mondolfo, quien, además, junto con su esposa Augusta Algranatte fueron los primeros en traducir, en 1968, Ciencia de la Lógica [1812] al español). Mucho más cerca en el tiempo, en buena parte de la Filosofia de la Liberación de Enrique Dussel (no tanto en las corrientes poscoloniales que suelen plantear un Hegel eurocéntrico y casi máxima expresión de la razón moderna). Finalmente, otro de los lugares en los que aparece esta relación Marx-Hegel es en el posmarxismo. La polémica entre Slvoj Zizek, Judith Butler y Ernesto Laclau, publicada en Contingency, Hegemony, Universality: Contemporary Dialogues OnTheLeft (2000), se relaciona, en cierta medida, con esta inclusión de Hegel entre Marx y Lacan.

La obra de Dri (2019) asume la premisa leninista sobre la relación entre Hegel y Marx y avanza en una hipótesis que pone en el centro a la dialéctica. Sin rodeos, nuestro autor afirma: "La dialéctica no es otra cosa que el movimiento lógico de la realidad en el sentido fuerte, la verdadera realidad (Wirklichkeit), la del sujeto, de los sujetos, de la intersubjetividad o del espíritu (p. 15).

Este posicionamiento coloca a la dialéctica como una ontología y no como un método, aunque la posición ontológica tendrá influencias metodológicas. Esta ontología dialéctica es la que asume la contradicción como inherente. El tema de la contradicción dialéctica ha sido un tema mal comprendido y ha derivado en una una disímil serie de conocidas objeciones como las elaboradas por Karl Popper, Isaiha Berlin, Galvano Della Volpe y Lucio Coletti, entre otros. Ahora bien, como han mostrado los estudiosos de Hegel, no existe en su obra algo como una negación del principio formal de no-contradicción. La contradicción dialéctica no es una contradicción lógica formal del tipo A y -A sino la inclusión de la negatividad (o de la nada) en el terreno del ser. Esta negatividad es parte del devenir del ser, que se constituye en singular mediante la negación del universal y luego la negación del particular resultante de recuperar la universalidad. Por eso, la realidad es tanto una negatividad como una positividad.

En Fenomenología del Espíritu (2006, [1807]), Hegel expone la experiencia de la conciencia que parte de considerar -ingenuamente- que el conocimiento sensible es el más "concreto" y verdadero. Sin embargo, lo que se presenta 
como verdadero e inmediato (el esto para el este) es el conocimiento más abstracto y pobre. Dri (2019) recuerda que en Hegel esto tiene un doble efecto. Por un lado, una lección metodológica, pero, por otro, una antropológica, en tanto en la búsqueda de la certeza sensible lo que emerge es el problema del sujeto o de la conciencia. En ambos planos aparece la intersubjetividad. En el metodológico, porque no hay posibilidad de trascender el mero conocimiento sensorial sin presuponer el mundo intersubjetivo (el lenguaje). En lo antropológico, porque no hay autoconciencia sin la presuposición del otro y su reconocimiento. En este segundo aspecto es donde aparece una lucha por el reconocimiento (el célebre pasaje sobre señorío y servidumbre de la Fenomenología).

En efecto, Dri (2019) sostiene que el pasaje de Fenomenología del Espíritu (1807) que más impresionó a Marx fue el de la lucha por el reconocimiento. La recuperación de la cuestión de la negatividad y del trabajo es algo que Marx, según Dri (2019), mantendrá durante el resto de su vida. En la constitución del sujeto y de la subjetividad, ambos aspectos (negatividad y trabajo) serán constitutivos. La preocupación de Marx por las condiciones de "devenir sujetos" en el capitalismo le permitió postular la idea de una realización enajenada y, desde allí, pensar tanto la crítica a esta situación como atisbar los escenarios de su superación.

\section{Dialéctica:}

\section{lógica ontológica y método}

Volvamos a la definición axiomática del libro: "La dialéctica no es otra cosa que el movimiento lógico de la realidad en sentido fuerte" (Dri, 2019, p. 15). Más allá de un debate si asumimos que la realidad es dialéctica (digamos independiente del sujeto) o si la ponemos como dialéctica, podemos conceder que la historia es dialéctica porque no es otra cosa que el despliegue mediante contradicciones (también históricas) y por lo tanto no deterministas. Dirá Dri (2019): "Como todo el proceso es dialéctico y, como sabemos, la dialéctica no es determinista, dicho proceso siempre está abierto a nuevas intervenciones" (p. 156). La idea de Hegel preso de una teleología no es más que un mito. 
Dri (2019) lo expresa del siguiente modo: "No hay determinaciones (Bestimmheit) sino condicionamiento (Bedingung). Si existiera determinación no habría dialéctica" (p.70). Es esta la enseñanza que retoma Marx.

En el capítulo 5, "Hegel: la idea absoluta", Dri (2019) trabaja de un modo excelso el vínculo metodológico entre Hegel y Marx mediado por la dialéctica. También lo hace en la sección 2 del capítulo 6, en la que revisa "El método en la economía política", que a su vez es iluminado por la interpretación de la tesis II sobre Feuerbach. Morfogénesis de la historia, lógica de la construcción del objeto y criterio de verdad condensados en un tratamiento sintético y eficaz.

Es en este punto en el que Marx, a partir de la crítica al idealismo, sienta las bases de una metodología para la construcción del objeto, en el sentido de método como camino dialéctico. El punto de partida parece el mismo: lo abstracto, pero Marx complejiza el asunto. Por un lado, porque el materialismo (en su versión feuerbachiana) sería la espera de información mediante la intuición sensible de lo dado y, en este sentido, sería prekantiana. Por otro, porque esa realidad ante los sentidos no está dada sino puesta por la historia. En la epistemología de las ciencias sociales contemporáneas esto constituye una doble dimensión del constructivismo.

El corolario para el nuevo materialismo es recuperar la dimensión activa del sujeto tanto como productor de la realidad como en su dimensión de producción de conocimiento (algo que había quedado en manos del idealismo). Al idealismo, sin embargo, le cuestiona desatender la dimensión creativa del sujeto en relación con la realidad. En este sentido, "sujeto y objeto, condiciones subjetivas y objetivas, hombres y circunstancias, lo puesto y lo presupuesto juegan indefinidamente originando diversos momentos históricos" (Dri, 2019, p. 70). La superación de la dicotomía idealismo-materialismo, como puede apreciarse, encuentra su umbral. La negatividad es constitutiva porque es preciso negar ese particular en el universal para construir el objeto singular. Objeto que nos permite reconstruir las determinaciones internas de un proceso social, a la vez que nos dice más que del mero caso particular.

En términos metodológicos, partimos de la universalidad abstracta, pero para encontrar sus determinaciones es necesario un doble movimiento. Por un lado, comprender que las determinaciones que hacen de "esto" un "esto" son procesos históricos inescindibles de la praxis humana ( $\mathrm{y}$, por lo tanto, 
construidos). En Ciencia de la Lógica (1968 [1812]), Hegel sostiene que "las determinaciones del pensamiento y del concepto son aquellas en que el objeto es lo que es" (p. 732). Por otro, elaborar las distinciones conceptuales para la reconstrucción del proceso complejo. La célebre cita de Marx "si los hombres captasen inmediatamente las conexiones, ¿para qué serviría la ciencia?» (Marx a Engels, carta del 27-6-1867) o, también, "toda ciencia estaría de más si la forma de manifestarse las cosas y la esencia de éstas coincidiesen directamente» (Marx, 1965 [1867], p. 757).

Ahora bien, en la actualidad tendemos a sospechar de la existencia de esencias porque las asociamos a entidades inmutables, cuasinaturales y ahistóricas cuyo desvío es denunciado por los guardianes de la esencialidad. La crítica a la esencialización de los géneros, por ejemplo, ha sido una significativa contribución de las teorías feministas que, en cierto modo, desencializaron a la mujer para admitir pluralidades y disidencias identitarias. Sin embargo, esto no es contradictorio -justamente, lo contrario- si atendemos a lo que dice Marx y lo contextualizamos en su universo-temporal lingüístico. En la tesis VI (1845), Marx sostiene: "La esencia es el ensamble de relaciones sociales" (1981, p. 7), por lo que podemos pensar que esas esencias son sociales e históricas, por lo tanto, mutables y, a la vez, determinadas socialmente. Pero, además, esta definición tiene una función epistemológica: para conocer la esencia de una cosa es necesario poner el objeto (o construirlo), para estudiar las determinaciones internas que constituyen a un fenómeno como tal. Conocer el ensamble de relaciones sociales es conocer la esencia. La pregunta es si es posible desde esta metodologia pensar un método e, incluso, técnicas de estudio capaces de producir un conocimiento de las esencias.

Esta concepción produce una ruptura con el positivismo intuitivo. Por un lado, se abre un interrogante poco abordado por la epistemología: cómo pensar la negatividad (es decir lo que no-es) o lo que su existencia adquiere realidad cuando es negado. Este es el punto de partida metodológico de la Filosofia de la Liberación. Por otro, cómo pensar lo que puede ser y no es. Es decir, la dimensión de un futuro abierto que se concretiza a partir de la negación de la negación (como praxis de los sujetos en su devenir). Una de las claves está en la recuperación del criterio de verdad contenido en la Tesis II (1845), cuya traducción Dri (2019) expone: 
La pregunta (die Frage) de si al pensamiento humano se le puede atribuir una verdad objetiva no es una pregunta de la teoría, sino de la práctica. Es en la praxis donde el hombre tiene que demostrar la verdad, es decir, la realidad y el poder, la aquendidad de su pensamiento (p. 64).

Es evidente que esa "verdad" no remite a la correspondencia entre pensamiento y mundo externo. Para Dri (2019), verdad es realización porque la problemática interna del asunto tanto en Hegel como en Marx no está signada por una preocupación por la realidad intersubjetiva (humana). Estamos en el terreno para afirmar con Hegel (1968 [1812]) que "la Idea absoluta es ser, vida imperecedera, verdad que se conoce a sí misma, y es toda la verdad. La Idea absoluta es el único objeto y contenido de la filosofia" (p. 725). Pero la idea absoluta tiene diferentes configuraciones, modos de aprehensión de esa realidad: " $\mathrm{El}$ arte, la religión $\mathrm{y}$, finalmente la filosofia. Esta es la manera más elevada" (Dri, 2019, p. 77). Nótese lo que dice Marx (1992 [1843]) al respecto:

La totalidad que se manifiesta en la mente como un todo pensado es producto de la elaboración de los conceptos del cerebro penante que se apropia del mundo de la única manera posible. La apropiación práctica por el arte y la religión es enteramente diferente. En tanto que la mente posee una actividad puramente especulativa y teórica, el sujeto real subsiste de manera autónoma, aparte de la mente. Por eso que también en el método teórico, es preciso que el sujeto -la sociedad- obre constantemente sobre la mente como condición previa (p. 341).

Es preciso enfatizar, al menos, dos ideas de la anterior cita. La primera es la diferenciación de la estructura aprehensiva o comprensiva de la religión, el arte y la ciencia. Marx (1992 [1843]) no niega que la religión y la ciencia sean formas de apropiación práctica, sino que postula que son de otra índole. La segunda es que el sujeto es la sociedad y su existencia es ese entramada intersubjetivo, ese "ensamble de relacione sociales" que requiere de la praxis humana para constituirse, que se escinde y que se objetiva.

El método, entonces, no es la aplicación de un procedimiento a la realidad. Para Dri (2019), "no hay un método creado por el sujeto para aproximarse a la realidad. Es la misma realidad la que dicta el método, porque este no es otra 
cosa que su mismo movimiento. Pero estamos hablando de realidad como Wirklichkeit, es decir, como concepto o sujeto que es sujeto-objeto" (p. 79). En efecto, el método mismo es el movimiento de la realidad en que sujeto $\mathrm{y}$ objeto devienen dialécticamente desde el universal abstracto hasta el singular, pasando por el particular. El proceso de conocimiento nos lleva a concluir en un universal concreto que es resultante y que nos muestra las determinaciones que lo constituyen como singular. Comparemos cómo lo dice Hegel y cómo lo recupera Marx.

Dice Hegel (1968 [1812]):

La totalidad concreta, que constituye el comienzo, tiene, como tal, en ella misma el comienzo de su prosecución y su desarrollo. Como concreta, es distinta en sí; pero, a causa de su primera inmediación, los primeros distintos son, en primer lugar, diferentes. Sin embargo, lo inmediato, como universalidad que se refiere a sí misma, es decir, como sujeto, es también la unidad de estos diferentes. Esta reflexión es el primer grado del seguir adelante, es el surgir de la diferencia, es el juicio y el determinar en general. Lo esencial consiste en que el método absoluto halla y conoce la determinación de lo universal en este universal mismo. El conocer intelectual finito procede en esto de manera tal que acoge ahora de nuevo, e igualmente de modo extrínseco, los elementos del concreto que había dejado de lado en la generación abstractiva de aquel universal. Al contrario, el método absoluto no se comporta como reflexión extrinseca, sino que toma de su objeto mismo lo determinado, pues ella misma es el principio y el alma inmanentes de él (p. 730).

\section{Y recupera Marx (1992 [1843]):}

Lo concreto es concreto, ya que constituye la sintesis de numerosas determinaciones, o sea la unidad de la diversidad. Para el pensamiento constituye un proceso de sintesis y un resultado, no un punto de partida. Es para nosotros el punto de partida de la realidad y por tanto de la intuición y de la representación (...). Hegel cayó en la ilusión de concebir lo real como el resultado del pensamiento que se concentra en sí mismo, se profundiza y se mueve por sí mismo, mientras que el método que consiste en elevarse de lo abstracto a lo concreto es, para el pensamiento, la manera de apropiarse de lo concreto, o sea la manera de reproducirlo bajo la forma de lo concreto pensado (p. 340). 
En este proceso de conocimiento, Dri (2019) argumenta que es en Enciclopedia de las Ciencias Filosóficas (1817) donde Hegel es más claro al referir a los tres momentos: el abstracto (propio del entendimiento), el dialéctico o racionalnegativo y el especulativo o racional-positivo (p. 84). Marx (1992 [1843]), preocupado por la realidad de su tiempo, lo expresa, magistralmente, al argumentar que si comenzara su análisis por la población tendría una visión caótica del conjunto de relaciones que componen un modo de producción.

Si procediera mediante un análisis cada vez más penetrante arribaria a nociones cada vez más simples, partiendo de lo concreto que yo percibiera, pasaria a abstracciones cada vez más sutiles para desembocar en las categorias más simples (p. 339).

En Marx (1992 [1843]), aparece la doble inscripción de la dialéctica: como modo de poner la realidad y como el "método científico correcto" (p. 340). Ontología y metodologia, sujeto y objeto encuentran, de ese modo, su vínculo interno.

\section{A modo de cierre:}

la cuestión politica y el sujeto pueblo

La preocupación de Rubén Dri por la relación entre Marx y Hegel está lejos de ser una cuestión erudita. Es la política lo que ocupa y lo que preocupa a nuestro pensador. Sus esfuerzos por pensar la lógica de la liberación, la construcción del sujeto pueblo y su vínculo con la religión son una clara muestra de ello.

En el proceso dialéctico histórico lo concreto es lo que se concretiza; ello requiere de concebir el poder y el devenir sujeto histórico. En este momento es donde aparece la dimensión eminentemente política del asunto: el sujeto pueblo. La referencia de Lenin que abre y que cierra el libro también ubica esta preocupación central en la vida y en la obra de Rubén Dri. No se trata de una mera especulación filosófica como pudiera parecer si nos situáramos en el momento del universal abstracto sino que, mediado por la particular teoríapraxis, nos situamos en el momento del universal concreto, en este nivel: 
el pueblo. Ese sujeto pueblo, que despliega su poder, es pensado en su concreción histórica de muchas maneras. Esto obliga a repensar el vínculo entre lo histórico, lo universal y el devenir concreto de las experiencias singulares en América Latina.

Es cierto que si examinamos el modo de abordaje de la cuestión política en Hegel y en Marx encontramos que tienen proyectos diferentes. En este punto, Dri recupera la crítica al trabajo asalariado y la explotación propia de Marx (en este sentido, una crítica fuerte al capitalismo y a sus promesas humanizadas) con una apuesta al reconocimiento bajo alguna forma de religión (en el sentido que bien se explica) de re-ligar, es decir, una forma de restituir el lazo social, entre los individuos, entre los pueblos y como especie con la naturaleza.

Esa conformación del pueblo se plasma tanto como actor de la liberación (como afirmación de la negatividad y negación de la negación), como también en cuanto construcción de intersubjetividad (comunidad). Esta cuestión del pueblo como sujeto y su relación con la dialéctica es clave como momento del devenir -como dice Hugo Zemelman (1989)- la utopía en historia. Esta centralidad del sujeto pueblo, sus dimensiones míticas, su concreción en organizaciones populares y su proyecto son temas sobre los que Rubén Dri ha realizado aportes significativos a lo largo de su obra. Este debate adquiere nuevas condiciones en la América Latina actual, en los procesos de subsunción y marca los alcances de las respuestas populares.

\section{Referencias}

Borges, J. L. (1974). El Aleph. En Obras completas (1923-1972).

Ciudad Autónoma de Buenos Aires, Argentina: Emecé.

Butler, J., Laclau, E., Žižek, S. (2000). Contingency, Hegemony, Universality: Contemporary Dialogues on the Left. London, England: Verso. 
Dri, R. (1994). Razón y libertad. Hermenéutica del capitulo $V$ de Fenomenología del espíritu. Ciudad Autónoma de Buenos Aires, Argentina: Biblos.

Dri, R. (1998). La odisea de la conciencia moderna. Hermenéutica del capitulo VI de la Fenomenología del espíritu. Ciudad Autónoma de Buenos Aires, Argentina: Biblos.

Dri, R. (2002). Racionalidad, sujeto y poder. Irradiaciones de Fenomenología del espiritu. Ciudad Autónoma de Buenos Aires, Argentina: Biblos.

Dri, R. (2006). La Fenomenología del espiritu de Hegel. Perspectiva latinoamericana. Intersubjetividad y reino de la verdad (tomo 2). Ciudad Autónoma de Buenos Aires, Argentina: Biblos.

Dri, R. (2007). Hegel y la lógica de la liberación. La dialéctica del sujeto-objeto. Ciudad Autónoma de Buenos Aires, Argentina: Biblos.

Dri, R. (2009). La rosa en la cruz. La filosofía politica hegeliana. Ciudad Autónoma de Buenos Aires, Argentina: Biblos.

Dri, R. (2011). Hegelianas. Irradiaciones de la Fenomenología del espíritu. Ciudad Autónoma de Buenos Aires, Argentina: Biblos.

Dri, R. (2019). El movimiento dialéctico. De la Fenomenología del Espíritu de Hegel a los Grundrisse de Marx. Ciudad Autónoma de Buenos Aires, Argentina: Biblos.

Hegel, G. (1968) [1812]. Ciencia de la Lógica (Trad. de Augusta y Rodolfo Mondolfo). Ciudad Autónoma de Buenos Aires, Argentina: Solar.

Hegel, G. (1997) [1817]. Enciclopedia de las Ciencias Filosóficas (Trad. de Ramón Valls Plana). Madrid, España: Alianza. 
Hegel, G. (2006) [1807]. Fenomenología del Espiritu (Trad. de Manuel Jiménez Redondo). Valencia, España: Pre-textos.

Marechal, L. (1970). Megafón, o la guerra. Ciudad Autónoma de Buenos Aires, Argentina: Sudamericana.

Marx, C. (1965) [1867]. El Capital (tomo III) (Trad. de Wenceslao Roces). Ciudad de México, México: Fondo de Cultura Económica.

Marx, C. (1992) [1843]. La cuestión judia y otros escritos.

Ciudad Autónoma de Buenos Aires, Argentina: Planeta-Agostini.

Zemelman, H. (1989). De la historia a la politica. La experiencia de América Latina. Ciudad de México, México: Siglo XXI.

\section{Nota}

1 Este texto surgió a partir de mi participación en la presentación del libro de Rubén Dri, El movimiento dialéctico. De la Fenomenología del Espíritu de Hegel a los Grundrisse de Marx (Biblos, 2019), organizada por las facultades de Periodismo y Comunicación Social, y Humanidades y Ciencias de la Educación de la Universidad Nacional de La Plata el 26 de septiembre de 2019. Agradezco a Pablo Bilyk la oportuna invitación. 